\title{
On the Adaptive Integrand Decomposition of Two-loop Scattering Amplitudes
}

\author{
William J. Torres Bobadilla* $\dagger$ \\ Instituto de Física Corpuscular, Universitat de València - Consejo Superior de Investigaciones \\ Científicas, \\ Parc Científic, E-46980 Paterna, Valencia, Spain \\ E-mail: william.torres@ific.uv.es
}

\begin{abstract}
In this talk we review recent developments towards the calculation of multi-loop scattering amplitudes. In particular, we discuss their calculation at one- and two-loop level. This is done by means of the adaptive integrand decomposition algorithm. We show preliminary results on the analytic reduction of Einstein-Yang-Mills amplitudes at one-loop, as well as, the $\mu e$-elastic scattering at two-loop level.
\end{abstract}

Loops and Legs in Quantum Field Theory (LL2018)

29 April 2018 - 04 May 2018

St. Goar, Germany

\footnotetext{
* Speaker.

†Talk based on a collaboration with P. Mastrolia, G. Ossola, M. Passera, T. Peraro, A. Primo, J. Ronca, R. Sameshima and U. Schubert
} 


\section{Introduction}

In this talk, we discuss the calculation of scattering amplitudes from the perspective of integrand reduction methods $[1-4]$. We report the recent developments on the automation of the adaptive integrand decomposition algorithm (AIDA) [5, 6]. At one-loop level, we present, as an example, the evaluation of Einstein-Yang-Mills amplitudes. From this example, we emphasise that AIDA can also deal with integrands generated from non-renormalisable theories.

Furthermore, we study the analytical integrand reduction of the two-loop QED correction $\mu e$ elastic scattering [7, 8]. For this amplitude, AIDA provides the decomposition in terms of integrals, which can be reduced by integration-by-parts identities [9-11]. Then, as a final step, that will be presented elsewhere, we evaluate the master integrals, which were obtained in the companion papers [12, 13] and presented in this conference [14].

This contribution is organised as follows: in Sec. 2 we briefly describe the main ingredients of the adaptive integrand decomposition. We recap the decomposition of the space-time dimension into parallel and perpendicular components, $d=d_{\|}+d_{\perp}$. As well, the straightforward classification of spurious terms, and the usual polynomial division is replaced by algebraic substitutions. In Sec.s 3 and 4, we provide one- and two-loop examples in which AIDA has been applied on.

\section{Adaptive Integrand Decomposition in a nutshell}

In this section, we explain the main features of the Adaptive Integrand Decomposition Algorithm (AIDA), the automation of [5]. We remark that this method decomposes the space-time dimension, $d=4-2 \varepsilon$, into parallel (or longitudinal) and orthogonal (or transverse) dimensions, $d=d_{\|}+d_{\perp}$. Parallel and orthogonal directions show particular properties for topologies with less than five external legs.

In the structure of the Feynman integrals,

$$
\mathscr{I}_{i_{1} \cdots i_{n}}^{(\ell)}[\mathscr{N}]=\int\left(\prod_{i=1}^{\ell} \frac{d^{d} \bar{l}_{i}}{\pi^{d / 2}}\right) \frac{\mathscr{N}_{i_{1} \cdots i_{n}}\left(\bar{l}_{i}\right)}{\prod_{j} D_{j}\left(\bar{l}_{j}\right)}
$$

loop momenta become

$$
\bar{l}_{i}^{\alpha}=l_{\| i}^{\alpha}+\lambda_{i}^{\alpha}
$$

with

$$
\bar{l}_{\| i}^{\alpha}=\sum_{j=1}^{d_{\|}} x_{j i} e_{j}^{\alpha}, \quad \lambda_{i}^{\alpha}=\sum_{j=d_{\|}+1}^{4} x_{j i} e_{j}^{\alpha}+\mu_{i}^{\alpha}, \quad \lambda_{i j}=\sum_{l=d_{\|}+1}^{4} x_{l i} x_{l j}+\mu_{i j} .
$$

In Eq. (2.2), $l_{\| i}$ is a vector of the $d_{\|}$-dimensional space spanned by the external momenta, and $\lambda_{i}$ belongs the $d_{\perp}$-dimensional orthogonal subspace. In this parametrisation, all denominators become independent of the transverse components of the loop momenta.

Let us indicate with $\mathbf{z}$ the full set of $\ell(\ell+9) / 2$ variables

$$
\mathbf{z}=\left\{\mathbf{x}_{\| i}, \mathbf{x}_{\perp i}, \lambda_{i j}\right\}, \quad i, j=1, \ldots \ell,
$$


where $\mathbf{x}_{\| i}\left(\mathbf{x}_{\perp i}\right)$ are the components of the loop momenta parallel (orthogonal) to the external kinematics, the denominators are reduced to polynomials in the subset of variables

$$
\tau=\left\{\mathbf{x}_{\|}, \lambda_{i j}\right\}, \quad \tau \subset \mathbf{z},
$$

so that the general $r$-point integrand has the form

$$
\mathscr{I}_{i_{1} \ldots i_{r}}\left(\tau, \mathbf{x}_{\perp}\right) \equiv \frac{\mathscr{N}_{i_{1} \ldots i_{r}}\left(\tau, \mathbf{x}_{\perp}\right)}{D_{i_{1}}(\tau) \cdots D_{i_{r}}(\tau)}
$$

Since numerator and denominators depend on different variables, the adaptive integrand decomposition suggests the following algorithm:

1. Divide: we divide the numerator $\mathscr{N}_{i_{1} \ldots i_{r}}\left(\tau, \mathbf{x}_{\perp}\right)$ modulo the Gröbner basis $\mathscr{G}_{i_{1} \cdots i_{r}}(\tau)$ of the ideal $\mathscr{J}_{i_{1} \cdots i_{r}}(\tau)$ generated by the set of denominators. The polynomial division is performed be adopting the lexicographic ordering $\lambda_{i j} \ll \mathbf{x}_{\|}$,

$$
\mathscr{N}_{i_{1} \ldots i_{r}}\left(\tau, \mathbf{x}_{\perp}\right)=\sum_{k=1}^{r} \mathscr{N}_{i_{1} \ldots i_{k-1} i_{k+1} \ldots i_{r}}\left(\tau, \mathbf{x}_{\perp}\right) D_{i_{k}}(\tau)+\Delta_{i_{1} \ldots i_{r}}\left(\mathbf{x}_{\|}, \mathbf{x}_{\perp}\right) .
$$

The Gröbner basis does not need to be explicitly computed, since, with the choice of variables and the ordering described here, the division is equivalent to applying the set of linear relations described above.

2. Integrate: Since denominators do not depend on transverse variables, $\mathbf{x}_{\perp}$, we can integrate the residue $\Delta_{i_{1} \ldots i_{r}}$ over transverse directions. This integration is carried out by expressing $\Delta_{i_{1} \ldots i_{r}}$ in terms of Gegenbauer polynomials, i.e.,

$$
\Delta_{i_{1} \ldots i_{r}}^{\mathrm{int}}(\tau)=\int d^{\left(4-d_{\|}\right) \ell} \Theta_{\perp} \Delta_{i_{1} \ldots i_{r}}\left(\tau, \Theta_{\perp}\right)
$$

Where $\Delta_{i_{1} \ldots i_{r}}^{\mathrm{int}}$ is a polynomial in $\tau$ whose coefficients depend on the space-time dimension $d$.

3. Divide: the structure of the integrated residue suggests a second division. This can be seen from the dependence $\Delta_{i_{1} \ldots i_{r}}^{\text {int }}$ has on the variables $\tau$. In fact, after applying the division, similarly as in the first step of this algorithm, we get

$$
\Delta_{i_{1} \ldots i_{r}}^{\mathrm{int}}(\tau)=\sum_{k=1}^{r} \mathscr{N}_{i_{1} \ldots i_{k-1} i_{k+1} \ldots i_{r}}^{\mathrm{int}}(\tau) D_{i_{k}}(\tau)+\Delta_{i_{1} \ldots i_{r}}^{\prime}\left(\mathbf{x}_{\|}\right)
$$

where the new residue $\Delta_{i_{1} \ldots i_{r}}^{\prime}\left(\mathbf{x}_{\|}\right)$can only depend on $\mathbf{x}_{\|}$.

\section{One-loop Einstein-Yang-Mills amplitudes}

In this section, we show that AIDA can also deal with integrands generated from non-renormalised theories, e.g. gravity. For the purpose of the discussion, we focus on the Einstein-Yang-Mills theory.

Let us start with the Yang-Mills Lagrangian,

$$
\mathscr{L}_{\mathrm{YM}}=-\frac{1}{2} \sqrt{-g} g^{\mu \rho} g^{v \sigma} \operatorname{tr}\left[F_{\mu \nu} F_{\rho \sigma}\right]+\mathscr{L}_{\mathrm{YM} ; \mathrm{GF}}+\mathscr{L}_{\mathrm{FP}}
$$


and the Einstein-Hilbert Lagrangian,

$$
\mathscr{L}_{\mathrm{EH}}=\frac{2}{\kappa^{2}} \sqrt{-g} R+\mathscr{L}_{\mathrm{EH} ; \mathrm{GF}},
$$

whose sum,

$$
\mathscr{L}_{\mathrm{EYM}}=\mathscr{L}_{\mathrm{YM}}+\mathscr{L}_{\mathrm{EH}},
$$

leads to the Einstein-Yang-Mills Lagrangian. In Eq.s (3.1) and (3.2), $g$ is the complete metric with the Ricci scalar $R$ and $F_{\mu \nu}$ the field strength tensor, whereas the suffixes stand for "gauge fixing" and "Faddeev-Popov" terms.

In order to couple gravitons with gluons, we split the metric tensor $g$ in

$$
g_{\mu v}=\eta_{\mu v}+\kappa h_{\mu v}
$$

with $\eta_{\mu \nu}$ the flat Minkowski metric and $h_{\mu \nu}$ our graviton field. The coefficient $\kappa$ is the gravitational coupling constant, $\kappa=\sqrt{32 \pi} / M_{\text {Planck}}$. For a pedagogical derivation of the Feynman rules of this Lagrangian, we refer to $[15,16]$.

From the Lagrangian (3.3), we extract the Feynman rules needed to compute the EinsteinYang-Mills one-loop amplitudes, $A_{4}^{(1)}(1,2,3,4)$. In these proceedings we report only the divergent amplitude $A_{4}^{(1)}\left(1^{-}, 2^{-}, 3^{+}, 4^{++}\right)$, the other rational amplitudes with up to two gravitons have also been computed, finding agreement with Ref. [17].

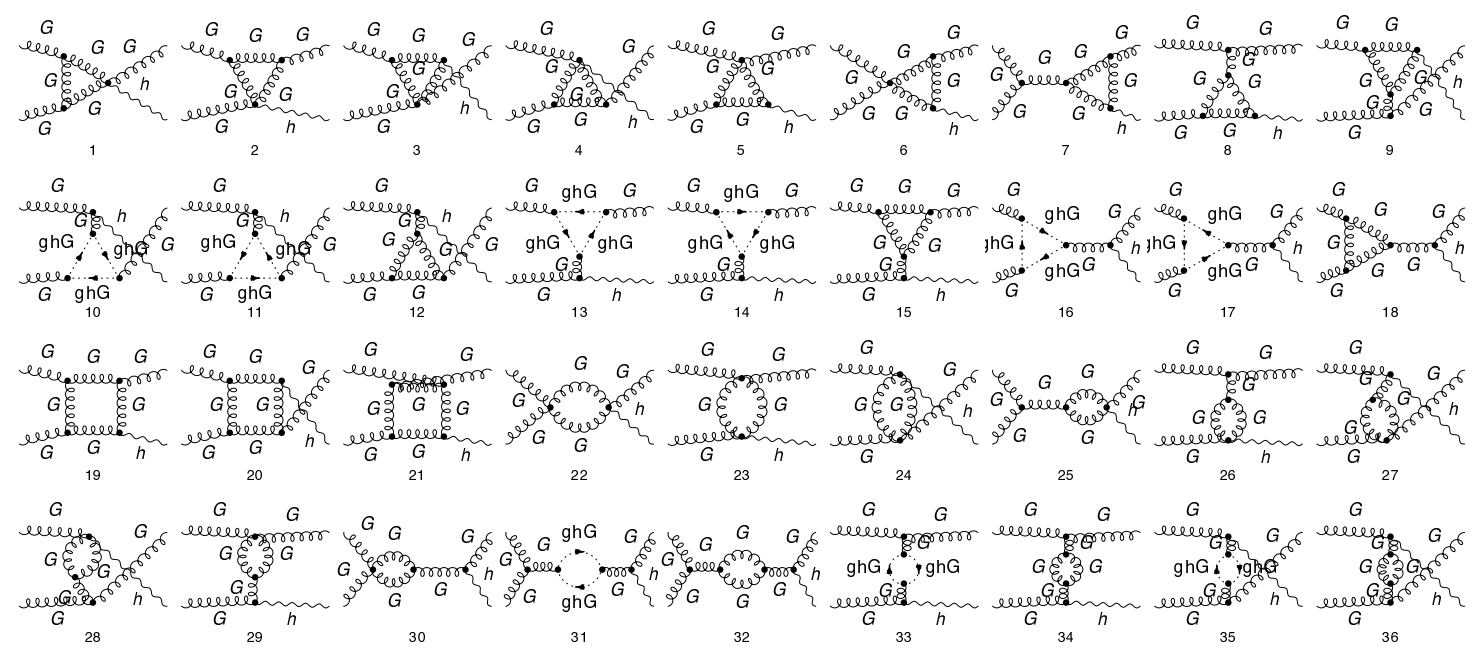

Figure 1: Feynman diagrams contributing to the Einstein-Yang-Mills one-loop amplitudes $A_{4}^{(1)}(1,2,3 ; 4)$.

Since we are interested in an approach based on first principles, we generate the integrands from the diagrams of Fig. 1 with the use of the MATHEMATICA packages FEYNARTs [18] and FEYNCALC $[19,20]$. Then, we follow the steps of AIDA:

1. Identify parent topologies and group diagrams: according to the numbering of diagrams of Fig. 1, we end up with three groups,

$$
\{\{19,1,2,5,6,7,8,13,14,15,16,17,18,22,23,25,26,29,30,31,32,33,34\},
$$


$\{20,3,4,9,10,11,12,24,27,28,35,36\},\{21\}\}$.

being the first element of each sublist the parent topology.

2. Generate cuts: the parent topology of the group 1,

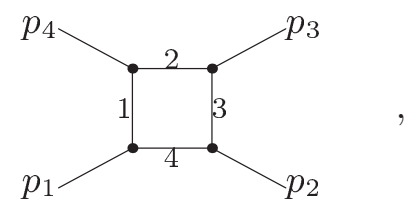

generates

$$
\{\{1,2,3,4\},\{2,3,4\},\{1,3,4\},\{1,2,4\},\{1,2,3\},\{2,4\},\{1,3\}\} .
$$

We remark that terms that vanish in dimensional regularisation, like massless tadpoles and bubbles, are never computed.

3. Define adaptive variables and prepare substitution rules for all cuts: for instance, the parametrisation of the diagram 22 becomes

$$
\begin{aligned}
& x_{1}^{(13)} \rightarrow \frac{d_{1}}{2}-\frac{d_{3}}{2}+\frac{1}{2}, \\
& \lambda_{11}^{(13)} \rightarrow-\frac{d_{1}^{2}}{4 t}+\left(\frac{d_{3}}{2 t}+\frac{1}{2}\right) d_{1}+\frac{d_{3}}{2}-\frac{d_{3}^{2}}{4 t}-\frac{t}{4} .
\end{aligned}
$$

4. Organise cuts in jobs: let us illustrate this organisation by considering the bubble contribution, cut $\{\{1,3\}\}$, to the amplitude coming from the first group:

$$
\left\{\mathbf{N}_{\{\{1,3\}}^{\{\{1,2,3\}}, \mathbf{N}_{\{\{1,3\}}^{\{\{1,3,4\}}, \mathbf{N}_{\{\{1,3\}}^{\{\{1,2,3\}}, \mathbf{N}_{\{\{1,3\}}^{\{\{1,3\}}\right\},
$$

where the first term corresponds to the bubble contribution coming from the reduction of the 4-point Feynman diagrams and the other contributions represent triangle and bubble reductions.

5. Divide: apply substitution rules of 3 to the numerator.

6. Collect powers of denominators to read off residue and numerators of lower cuts.

7. Integrate (substitute) transverse variables appearing in the residues.

8. Divide again, using as input numerators the residues.

After performing these steps, we obtain a decomposition in terms of one-loop scalar integrals, whose expansion in $d \rightarrow 4$, in the four-dimensional-helicity scheme, gives

$$
\begin{aligned}
A_{4}^{(1)}\left(1^{-}, 2^{-}, 3^{+} ; 4^{++}\right)= & A_{4}^{(0)} c_{\Gamma}\left(-\frac{\mu^{2}}{s}\right)^{\varepsilon}\left[-\frac{3}{\varepsilon^{2}}-\frac{11}{3 \varepsilon}-\frac{1}{\varepsilon}\left(\log \left(\frac{-s}{-t}\right)+\log \left(\frac{-s}{s+t}\right)\right)\right. \\
& -\frac{11}{3} \log \left(\frac{-s}{-t}\right)+\frac{t\left(14 s^{2}+9 s t+6 t^{2}\right)}{3 s^{3}} \log \left(\frac{-t}{s+t}\right)
\end{aligned}
$$




$$
\begin{aligned}
& +\left(\frac{t(s+t)\left(2 s^{2}+s t+t^{2}\right)}{s^{4}}+\frac{1}{2}\right)\left(\log ^{2}\left(\frac{-t}{s+t}\right)+\pi^{2}\right)+\pi^{2} \\
& \left.+\frac{t(s+t)}{s^{2}}-\frac{64}{9}\right] .
\end{aligned}
$$

\section{4. $\mu e$ elastic scattering}

Motivated by the new experiment MUonE proposed at CERN [21] that provides a new and independent determination of the leading hadronic contribution to the muon $g-2$ [21, 22], we consider as an application of AIDA, the two-loop reductions of the $\mu e$ elastic scattering,

$$
e^{-}\left(-p_{1}\right) \mu^{-}\left(-p_{4}\right) \rightarrow e^{-}\left(p_{2}\right) \mu^{-}\left(p_{3}\right)
$$

The electron is treated as massless, $m_{e}^{2}=0$, while we retain full dependence on the muon mass, $m_{\mu}^{2} \neq 0$. We focus on the spin summed/averaged squared matrix elements,

$$
M^{(1)}=2 \operatorname{Re}\left\langle A_{e \mu}^{(0)} \mid A_{e \mu}^{(1)}\right\rangle, \quad \quad M^{(2)}=2 \operatorname{Re}\left\langle A_{e \mu}^{(0)} \mid A_{e \mu}^{(2)}\right\rangle,
$$

to obtain the results of the corresponding two-loop amplitude.

Hence, we define the kinematical variables this amplitude depends on to be

$$
s=\left(p_{1}+p_{2}\right)^{2}, \quad t=\left(p_{2}+p_{3}\right)^{2}, \quad u=\left(p_{1}+p_{3}\right)^{2}=-s-t+2 m_{\mu}^{2} .
$$

For the calculation of the two-loop amplitude of the $\mu e$ elastic scattering, we have to evaluate the Feynman diagrams of [8, Fig. 3]. We follow the very same procedure as the one described in
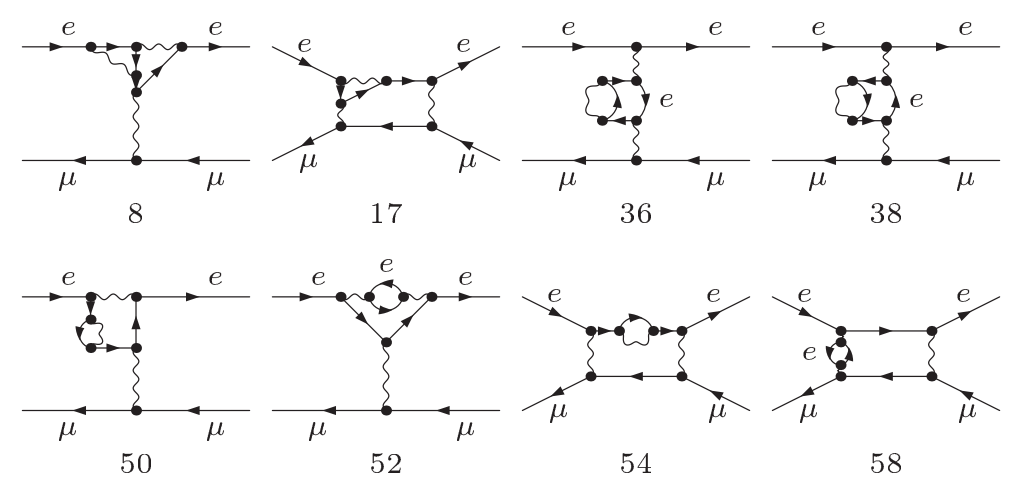

Figure 2: Selected Feynman diagrams contributing to the two-loop $\mu e$-scattering amplitude.

the previous section. Nevertheless, we describe the steps that could give a different interpretation

1. Identify parent topologies and group diagrams:

$$
\begin{aligned}
& \{\{1,46\},\{3,5\},\{6,35,41,67\},\{7\},\{16\},\{17,8,36,38,50,52,54,58\},\{18,60,65\},\{19\}, \\
& \{20\},\{21,14,51,56\},\{22\},\{23,57\},\{24,55,59\},\{25,2,4,9,11,13,34,47,66\}, \\
& \{26,10,12,15,37,39,40,43,44,48,68,69\},\{27\},\{28\},\{29,61,62\},\{30,64\},\{31\}, \\
& \{32,63\},\{33\},\{42\},\{45\},\{49\},\{53\}\} .
\end{aligned}
$$


2. Generate cuts: let us focus on the sixth group (see Fig. 2), whose parent topology

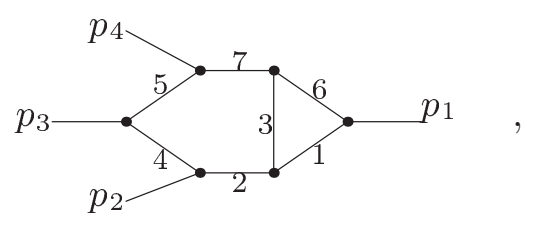

gives contribution to the following cuts

$$
\begin{aligned}
& \{\{\{1,6\},\{2,4,5,7\},\{3\}\},\{\{6\},\{2,4,5,7\},\{3\}\},\{\{1,6\},\{4,5,7\},\{3\}\},\{\{1,6\},\{2,4,5,7\}\}, \\
& \{\{1,6\},\{2,5,7\},\{3\}\},\{\{1,6\},\{2,4,7\},\{3\}\},\{\{1\},\{2,4,5,7\},\{3\}\},\{\{1,6\},\{2,4,5\},\{3\}\}, \\
& \{\{6\},\{4,5,7\},\{3\}\},\{\{6\},\{2,4,5,7\}\},\{\{6\},\{2,5,7\},\{3\}\},\{\{6\},\{2,4,7\},\{3\}\},\{\{2,4,5,7\}, \\
& \{3\}\},\{\{6\},\{2,4,5\},\{3\}\},\{\{1,6\},\{4,5,7\}\}, \ldots,\{\{1\},\{3\}\},\{\{1\},\{2\}\}\} .
\end{aligned}
$$

4. Organise cuts in jobs: in order to consider the full contribution of all possible diagrams, there is a subtlety w.r.t. the one-loop case, which relies on the treatment of the diagrams that contain squared propagators, e.g. diagrams 36-58 in Fig. 2. For instance, the contribution to the cut $\{\{1,6\},\{2,4,5\},\{3\}\}$ with linear propagators gets contributions from

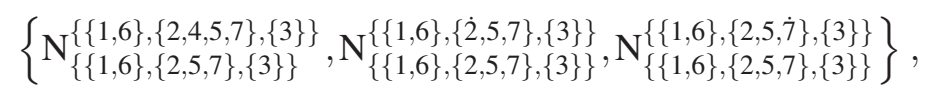

where dots represent squared propagators.

Unless the one-loop case, the two-loop result AIDA provides yet needs the evaluation of the Feynman integrals or reduce them to master integrals by means of integration-by-parts (IBPs) identities [9-11]. Nevertheless, the calculation of these master integrals has been carried out for the planar [12] and non-planar [13] (see references therein).

\section{Interface with IBP generators}

Since AIDA provides a decomposition in terms of Feynman integrals, which can be reduced to the so called master integrals, we also interface our results with the available IPB generators. To start with, we use REDUZE [23] and KIRA [24]. Nevertheless, the interface with many other IBP generators can be straightforwardly done.

In addition, the evaluation of (master) integrals is currently performed by SECDEC [25].

\section{Conclusions}

For the evaluation of one- and two-loop scattering amplitudes, we have reported on the progress of the implementation of the Adaptive Integrand Decomposition Algorithm (AIDA). This implementation automates, numerically and analytically, the method of [5]. We have also shown results for the analytic reduction of the one-loop Einstein-Yang-Mills amplitudes and two-loop QED correction to the $\mu e$-elastic scattering.

We have also interfaced our results with the integration-by-parts identities generators, as well as the evaluation of the (master) integrals. 


\section{Acknowledgments}

We are grateful to the Mainz Institute for Theoretical Physics (MITP) for its hospitality and support during the workshop "The evaluation of the leading hadronic contribution to the muon anomalous magnetic moment". This work has been supported in part by Grants No. FPA201453631-C2-1-P, FPA2017-84445-P and SEV-2014-0398 (AEI/ERDF, EU).

The Feynman diagrams depicted in this paper were generated using FEYNARTs [18].

\section{References}

[1] G. Ossola, C. G. Papadopoulos and R. Pittau, Reducing full one-loop amplitudes to scalar integrals at the integrand level, Nucl. Phys. B763 (2007) 147-169, [hep-ph / 0609007$].$

[2] P. Mastrolia, E. Mirabella and T. Peraro, Integrand reduction of one-loop scattering amplitudes through Laurent series expansion, JHEP 06 (2012) 095, [1203. 0291].

[3] S. Badger, H. Frellesvig and Y. Zhang, An Integrand Reconstruction Method for Three-Loop Amplitudes, JHEP 1208 (2012) 065, [1207.2976].

[4] P. Mastrolia, E. Mirabella, G. Ossola and T. Peraro, Integrand-Reduction for Two-Loop Scattering Amplitudes through Multivariate Polynomial Division, Phys. Rev. D87 (2013) 085026, [1209.4319].

[5] P. Mastrolia, T. Peraro and A. Primo, Adaptive Integrand Decomposition in parallel and orthogonal space, JHEP 08 (2016) 164, [1605.03157].

[6] P. Mastrolia, T. Peraro, A. Primo and W. J. Torres Bobadilla, Adaptive Integrand Decomposition, PoS LL2016 (2016) 007, [1607. 05156].

[7] P. Mastrolia, M. Passera, A. Primo, U. Schubert and W. J. Torres Bobadilla, On $\mu$ e-scattering at NNLO in QED, EPJ Web Conf. 179 (2018) 01014.

[8] W. J. Torres Bobadilla, Interplay of colour kinematics duality and analytic calculation of multi-loop scattering amplitudes: one and two loops, PoS RADCOR2017 (2018) 082, [1801.03010].

[9] F. V. Tkachov, A Theorem on Analytical Calculability of Four Loop Renormalization Group Functions, Phys. Lett. B100 (1981) 65-68.

[10] K. G. Chetyrkin and F. V. Tkachov, Integration by Parts: The Algorithm to Calculate beta Functions in 4 Loops, Nucl. Phys. B192 (1981) 159-204.

[11] S. Laporta, High precision calculation of multiloop Feynman integrals by difference equations, Int. J. Mod. Phys. A15 (2000) 5087-5159, [hep-ph/ 0102033$].$

[12] P. Mastrolia, M. Passera, A. Primo and U. Schubert, Master integrals for the NNLO virtual corrections to $\mu$ e scattering in QED: the planar graphs, JHEP 11 (2017) 198, [1709. 07435].

[13] S. Di Vita, S. Laporta, P. Mastrolia, A. Primo and U. Schubert, Master integrals for the NNLO virtual corrections to $\mu$ e scattering in QED: the non-planar graphs, 1806.08241.

[14] A. Primo, Two-loop integrals for $\mu$ e scattering, These Proceedings (2018).

[15] A. Rodigast, One-Loop Divergences of the Yang-Mills Theory Coupled to Gravitation, Master's thesis, Diploma thesis, 2007, Humboldt University Berlin, available at http://qft.physik.hu-berlin.de., 2008.

[16] T. Schuster, Lee-Wick Gauge Theory and Effective Quantum Gravity, Master's thesis, Diploma thesis, 2008, Humboldt University Berlin, available at http://qft.physik.hu-berlin.de., 2008. 
[17] D. Nandan, J. Plefka and G. Travaglini, All rational one-loop Einstein-Yang-Mills amplitudes at four points, 1803.08497.

[18] T. Hahn, Generating Feynman diagrams and amplitudes with FeynArts 3, Comput. Phys. Commun. 140 (2001) 418-431, [hep-ph / 0012260$].$

[19] R. Mertig, M. Bohm and A. Denner, FEYN CALC: Computer algebraic calculation of Feynman amplitudes, Comput. Phys. Commun. 64 (1991) 345-359.

[20] V. Shtabovenko, R. Mertig and F. Orellana, New Developments in FeynCalc 9.0, 1601 . 01167.

[21] G. Abbiendi et al., Measuring the leading hadronic contribution to the muon g-2 via $\mu$ e scattering, Eur. Phys. J. C77 (2017) 139, [1609.08987].

[22] C. M. Carloni Calame, M. Passera, L. Trentadue and G. Venanzoni, A new approach to evaluate the leading hadronic corrections to the muon g-2, Phys. Lett. B746 (2015) 325-329, [150 4 . 02228].

[23] A. von Manteuffel and C. Studerus, Reduze 2 - Distributed Feynman Integral Reduction, 1201.4330 .

[24] P. Maierhöfer, J. Usovitsch and P. Uwer, Kira-A Feynman integral reduction program, Comput. Phys. Commun. 230 (2018) 99-112, [1705 . 05610].

[25] S. Borowka, G. Heinrich, S. P. Jones, M. Kerner, J. Schlenk and T. Zirke, SecDec-3.0: numerical evaluation of multi-scale integrals beyond one loop, Comput. Phys. Commun. 196 (2015) 470-491, [1502.06595]. 